\title{
Registration and Integration for Fluoroscopy Device Enhancement
}

\author{
James C. Ross ${ }^{1}$, David Langan ${ }^{1}$, Ravi Manjeshwar ${ }^{1}$, John Kaufhold ${ }^{1}$, \\ Joseph Manak ${ }^{1}$, and David Wilson ${ }^{2}$ \\ ${ }^{1}$ GE Research Center, Niskayuna NY 12309, USA \\ 2 Case Western Reserve University, Cleveland OH 44106, USA
}

\begin{abstract}
We investigated a method, motion compensated integration (MCI), for enhancing stent Contrast-to-Noise Ratio (CNR) such that stent deployment may be more easily assessed. MCI registers fluoroscopic frames on the basis of stent motion and performs pixel-wise integration to reduce noise. Registration is based on marker balls, high contrast interventional devices which guide the clinician in stent placement. It is assumed that stent motion is identical to that of the marker balls. Detecting marker balls and identifying their centroids with a high degree of accuracy is a non-trivial task. To address the required registration accuracy, in this work we examine MCI's visualization benefit as a function of registration error. We employ adaptive forced choice experiments to quantify human discrimination fidelity. Perception results are contrasted with CNR measurements. For each level of registration inaccuracy investigated, MCI conferred a benefit $(p<0.05)$ on stent deployment assessment suggesting the technique is tolerant of modest registration error. We also consider the blurring effect of cardiac motion during the x-ray pulse and select frames for integration as a function of cardiac phase imaged.
\end{abstract}

\section{Introduction}

Coronary artery disease (CAD) afflicts approximately thirteen million Americans [1. It is caused by the buildup of plaques and fatty deposits on the interior walls of coronary arteries. These narrowed (stenotic) vessels restrict blood flow to the heart and can ultimately lead to myocardial infarction. One of the standard techniques used to restore function to an impaired coronary vessel is stent deployment. Using x-ray fluoroscopy, the operating cardiologist guides a stent, compressed on a guidewire, through a catheter in the patient's vasculature to the site of the stenosis. The position of the stent on the guidewire is delineated by two radio-opaque marker balls. The marker balls serve as high contrast visual cues in the x-ray imagery for the clinician to position the stent prior to deployment. Once the stent is positioned to span the site of the stenosis, the balloon is inflated to expand the stenotic vessel and simultaneously deploy the stent. Figure 1 depicts an example fluoroscopic image containing a catheter, guidewire, marker balls, stent, and balloon. The deployed stent is often barely perceptible, even with the visual cues provided by the marker balls, making it difficult to ascertain whether the stent has been fully deployed. Partial deployment occurs when some portions of the stent fail to fully expand. This is problematic 


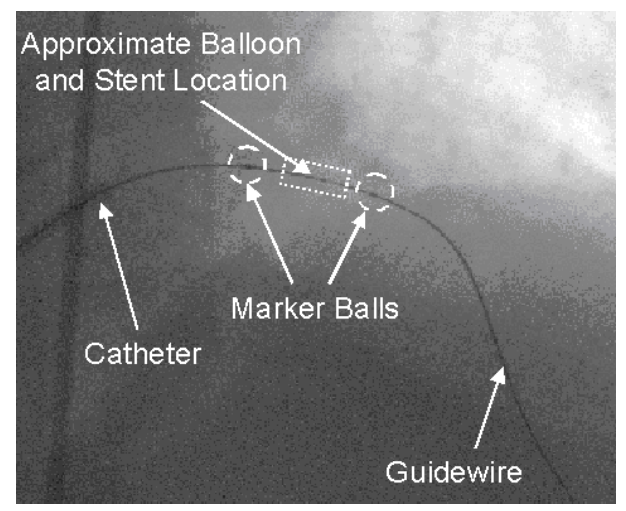

Fig. 1. Sample fluososcopic image. The marker balls on the guidewire assist in visualizing the stent location.

since stent malapposition is believed to increase the chance of restenosis. In an effort to determine whether a stent is completely deployed, a cardiologist will typically administer a bolus of contrast agent to visualize the flow through the stented vessel via fluoroscopy. A uniform flow profile along the length of the stent indicates proper dialation of the stenosis and can be used to infer full stent deployment. In some cases the ends of an otherwise fully deployed stent are "pinched" (i.e. improperly deployed) while the flow profile of the bolus suggests successful deployment. The flow profile of a contrast bolus is an indirect and incomplete measurement of stent deployment. There exists a clinical need for direct assessment of stent deployment.

We overview a method, motion compensated integration (MCI), for enhancing the stent CNR so that stent deployment may be assessed directly. Here, CNR is defined as the difference between the stent mean intensity and the background mean intensity divided by the background noise standard deviation. The background noise standard deviation is computed over a flat image region. The method parallels the approach taken by Koolen [2]. In this work we aim to measure the benefit of applying MCI to fluoroscopic x-ray sequences as a function of registration accuracy. We measure MCI performance in terms of human perception studies and contrast these results with computed CNR. These results may be used to specify registration performance. In section 2 we survey device properties, common imaging geometries and techniques leading to expected device CNR. In section 3 we briefly overview our method for improving stent CNR. In sections 5 through 7 we present our experimental approach, results, and discussion. Finally, in section 8 conclusions will be drawn.

\section{Devices, Acquisition Geometries, and Expected CNRs}

In order to ascertain the proper deployment of a stent, the shape of the stent must be resolved. In a projection x-ray image of a stent, multiple struts often 
Table 1. CNR Values for Patient Thicknesses and System Parameters

\begin{tabular}{|c|c|c|c|c|c|}
\hline Patient Thickness $(\mathrm{cm})$ & $\mathrm{kVp}$ & $\mathrm{mAs}$ & Cu Filtration (um) & Marker Ball CNR & Stent CNR \\
\hline 20 & 75 & 1.1 & 200 & 9.21 & 2.57 \\
25 & 85 & 0.767 & 200 & 5.20 & 1.31 \\
30 & 120 & 0.303 & 200 & 2.75 & 0.56 \\
35 & 120 & 0.303 & 200 & 1.54 & 0.31 \\
40 & 120 & 0.303 & 200 & 0.87 & 0.19 \\
\hline
\end{tabular}

occupy the same ray path from source to detector. Here, as a baseline, we will determine the CNR of an individual stent strut for a range of common patient thicknesses subject to typical x-ray system performance for fluoroscopy. Stent struts are commonly made of thin $(\sim 0.16 \mathrm{~mm})$ wires of a cobalt-chromium or stainless steel alloy. In the table below are shown the calculated CNRs for a $0.16 \mathrm{~mm}$ thick cobalt-chromium stent strut and a $1.0 \mathrm{~mm}$ Tungsten markerball for a variety of patient thicknesses. The simulated X-ray system was operating in fluroscopic mode $(<10 \mathrm{Rad} / \mathrm{min})$ filtered by a 200 micron layer of copper at a frame rate of 30 frames per second $(20 \mathrm{~cm}$ FOV, $70 \mathrm{~cm}$ SOD, $90 \mathrm{~cm}$ SID). The background noise term was calculated based on the quantum noise and an electronic noise term to simulate a digital flat-panel detector. As can been seen from the table, there is an order of magnitude variation in stent and markerball CNR as a function of projected patient thickness.

\section{Motion Compenstated Integration (MCI)}

The objective of MCI is to reduce the quantum noise in the x-ray image while retaining stent contrast. The strategy is to take a series of x-ray images, co-register the frames on the basis of stent location, and average the frames. We assume the stent motion is rigid to that of the markerballs and that the markerballs are present in each of the x-ray images. Due to their relatively high CNR, the markerballs are more easily detected and are used as a surrogate motion estimate for the stent. Each frame in the fluoroscopic sequence is registered to the first frame on the basis of the markerball centroids using a similarity transform 3 .

X-ray frames with significant out of plane motion, identified by a foreshortening of the distance between the markerballs may be ignored. Similarly, frames exhibiting significant blurring, identified by ECG or extended markerball footprint, may also be discarded.

The quantum x-ray noise is modelled as a Poisson process. For regions with constant mean, the noise may be equivalently modelled as an independent, identically distributed (i.i.d.) Gaussian process. It is assumed that for a given pixel location, the mean is constant among the registered frames. Therefore, the noise may be temporally averaged resulting in a noise reduction by $\sqrt{N}$, where $N$ is the number of frames averaged.

Once the sequence frames have been registered to the reference frame, they are temporally averaged according to the following expression 


$$
\mathbf{M C I}=\frac{1}{\mathbf{n}+\mathbf{1}}\left[\mathbf{I}_{\mathbf{0}}+\sum_{\mathbf{i}=\mathbf{1}}^{\mathbf{n}} \mathbf{T}_{\mathbf{i}}\left(\mathbf{I}_{\mathbf{i}}\right)\right]
$$

where $n+1$ is the number of frames in the sequence, $\mathbf{I}_{\mathbf{0}}$ is the reference frame, $\mathbf{I}_{\mathbf{i}}$ is the $i^{\text {th }}$ frame in the sequence, and $\mathbf{T}_{\mathbf{i}}$ is the transformation that registers frame $\mathbf{I}_{\mathbf{i}}$ to the reference frame. The result is referred to as the motion compensated integration image, MCI.

\section{Marker Ball Detection and Tracking}

A critical issue for MCI to be routinely used in a catheterization laboratory is automated unsupervised detection and tracking of the marker balls. This topic is beyond the scope of this paper, and here we briefly outline our dynamic programming based approach for marker ball detection and tracking.

The first step is to design a matched filter for a single marker ball on the basis prior knowledge of the device and acquisition geometry. Detecting the marker balls individually avoids the need to address the orientation of marker ball pairs.

The marker ball matched filter is applied to each frame. The processed frame is then thresholded for a specified constant false alarm rate, $N$. The $N$ detections are paired in accordance with a distance criterion determined by the device geometry and imaging magnification factor. Detects which are not successfully paired are discarded. Paired detects are tracked through multiple frames on the basis of ECG with an allowance for respiratory motion. Paired detects which fail to track are discarded. We further discard detects whose motion deviates from an ECG periodicity assumption. After a small number of cardiac cycles, the marker balls are identified and tracked through the frames.

\section{Experimental Methods}

The objective of our work was to quantify the benefit of MCI, as a function of registration accuracy, relative to the standard fluoroscopic sequence for stent deployment assessment. We employed a four alternative forced-choice (4-AFC) discrimination paradigm to measure the necessary stent CNR for the observer to correctly assess stent deployment 90 percent of the time 4] for both our baseline fluoroscopic sequence and for MCI subject to a specified registration accuracy.

At a high level, AFC experiments involve visually discriminating between two objects, a reference object and a test object, fully and partially deployed stents respectively. For a given trial, an observer is presented with four images one contains the partially deployed stent. The contrast with which the stents are displayed is adjusted over the course of many trials such that, at the experiment's conclusion, the contrast level for which the observer achieves 90 percent detection accuracy of the test object is reliably identified.

The AFC experiments required a database of fluoroscopic sequences and stent templates. We created a database of clinically acquired sequences that had a relatively uniform level of noise and background clutter. 
Next, we digitally added a guidewire with marker balls to each database sequence with periodic elliptic interframe motion. A $0.75 \mathrm{~mm}$ (3 pixel) guidewire with $1.5 \mathrm{~mm}$ (6 pixel) marker balls was synthetically generated and low pass filtered to mimic detector MTF. Our fully deployed stent template was based on an x-ray image of a Cordis drug-eluting stent with $160 \mu$ strut diameter. This stent template was manipulated by downsampling both ends to simulate partial deployment. The intensity of the downsampled ends was adjusted to maintain the level of total x-ray attenuation by the stent.

Registration error was modelled in polar coordinates with uniform distribution in $\theta$ and Gaussian, $\mathrm{N}\left(0, \sigma^{2}\right)$, in $r$. We measured MCI performance for $\sigma=[0.25,0.75,1.25,2.0,4.0]$.

We followed the reference/test technique described in [4] to conduct our experiments. The stent contrast was adapted so as to maintain observer performance at $90 \%$ probability of making the correct choice corresponding to a detectability index, $d^{\prime}$, of 2.4517 [5]. Results were obtained after 100 reference trials interlaced with 100 test trials. Contrast levels were determined from maximumlikelihood estimates, and standard errors were estimated by a method that accounted for adaptation. We used the Michelson contrast for our experiments:

$$
C=\frac{\left|\mu_{b}-\mu_{s}\right|}{\mu_{b}+\mu_{s}}
$$

where $\mu_{b}$ and $\mu_{s}$ were the mean gray-scale value of the background and stent, respectively. The normalized stent templates were added to the sequences according to the following expression:

$$
\mathbf{A}^{\prime}=\mathbf{A}-\frac{2 \mu_{\mathbf{b}} \mathbf{C}}{1+\mathbf{C}}(\mathbf{1}-\mathbf{S})
$$

where $\mathbf{A}$ is the anatomical sequence, $\mathbf{S}$ is the stent template sequence, and $\mathbf{A}^{\prime}$ is the resultant sequence.
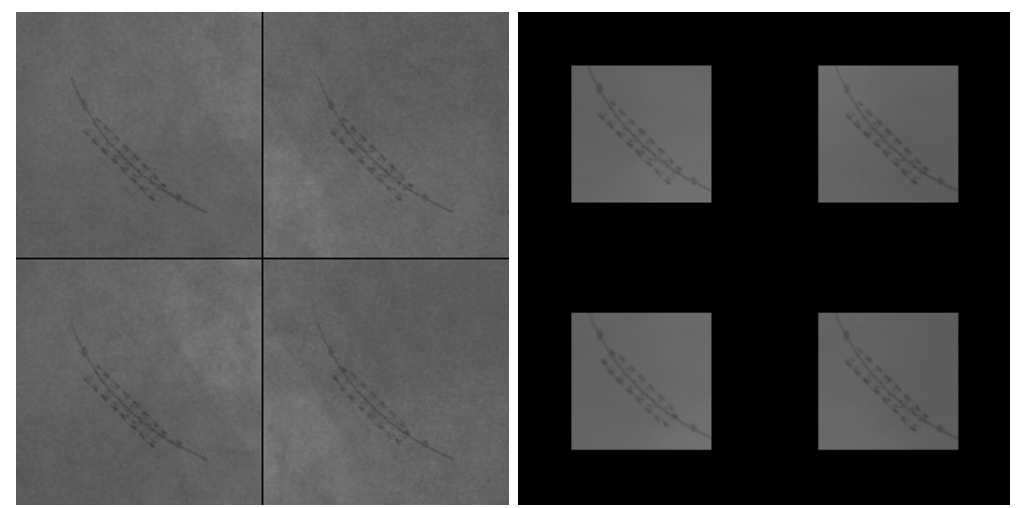

Fig. 2. Left: Example reference experiment trial. Right: Example test experiment trial. The stents were added to the fluoroscopy sequences with a high level of contrast for display purposes. 
For the reference experiment, four sequences were randomly selected from the database, and stent templates (three fully deployed, one partially deployed) were added to them according to (3). They were then simultaneously displayed on a medical quality gray-scale monitor with the location of the test sequence randomly varied. The observer selected the sequence most likely to contain the partially deployed stent.

Each reference trial was followed by a test trial. Interlacing the reference (fluoroscopic sequence) and test (MCI) trials minimized observer effects such as fatigue, lapses in attention, or possible physiological changes that could occur during the experiments [4. Figure 2 shows example reference and test trials.

\section{Results}

Contrast sensitivity is defined as the inverse of the final Michelson contrast values following adaptation. A higher sensitivity indicates an improved ability to detect the partially deployed stent. The ratio of test sensitivity to reference sensitivity for a given experiment is used in order to compensate for inter-observer variation. A ratio greater than one indicates an improved discrimination ability using MCI.
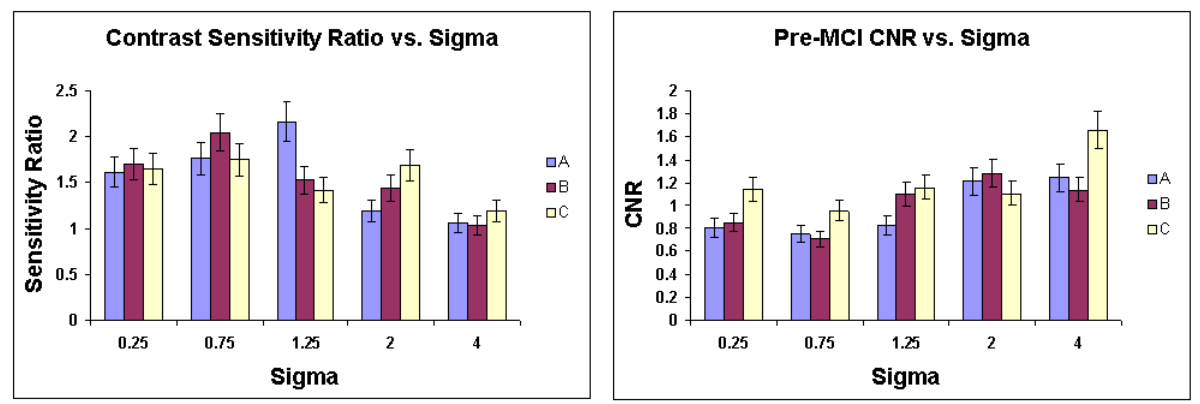

Fig. 3. Left: contrast sensitivity results for observers A, B, and C. Right: pre-MCI CNR results for observers $\mathrm{A}, \mathrm{B}$, and $\mathrm{C}$.

Figure 3 gives contrast sensitivity ratios for each observer. A one-sample ttest shows that MCI provides the observer a significant $(p<0.05)$ advantage for performing the discrimination task for each sigma value investigated. There is not a statistically significant trend over sigma values of $0.25,0.75$, and 1.25 , but there is a fall-off in contrast ratio levels exceeding 1.25. The decrease in ratios over this range indicates the blurring due to registration error is impacting perception. Figure 3 also shows pre-MCI CNR values for each observer (corresponding to test experiment final contrast values). It is reassuring to see that these values are representative of what we can expect to observe as presented in table 1 


\section{Discussion}

MCI enhances the visibility of stents through two mechanisms: the integration of multiple image frames to reduce noise; and removing motion enabling the observer to inspect a spatially static stent. It has been shown that the spatiotemporal bandwith of the human visual system degrades the detection of low contrast targets that are moving [6]. Whiting et al have shown that the detection of vessel defects is enhanced when the vessels are stabilized 7 .

MCI performance degrades with registration error, but more slowly than perhaps expected. Although a stent strut width is on the order of one pixel, the projected stent scaffolding width often spans several pixels. This multi-pixel projection footprint coupled with the knowledge that the observer is concerned with the overall shape of the stent, not individual struts, leads to the expectation that a sub-pixel registration accuracy is not required. Indeed, our perception studies shows MCI to be beneficial for a registration error sigma up to a value of 2 (the benefit of MCI at a sigma value of 4 is marginal). Given that a marker ball may be reliably detected, and it's radius is typically 3 pixels, a registration error sigma of 2 should be achievable. This alone motivates investigation of real-time unsupervised markerball detection and tracking approaches.
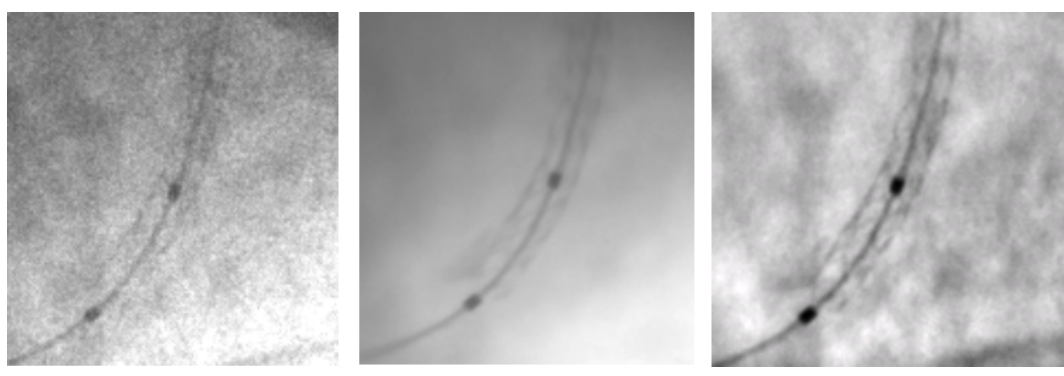

Fig. 4. MCI enhancement applied to a clinical sequence. Left: frame taken from original sequence. Middle: all fluoroscopy frames used for enhancement. Right: frames with motion blurring omitted from integration.

MCI has two opportunities to improve upon the perception performance reported. First, more frames may be used in the integration. However, noise decreases at a rate of $\sqrt{N}$, so the law of diminishing marginal returns applies. Second, we made no attempt to manipulate the displayed contrast of the MCI image relative to the standard fluoroscopic sequence. However, the opportunity to increase the displayed contrast of the MCI image exists as a result of the significantly reduced noise. Finally, we need to be cautious in that cardiac motion during the x-ray pulse can significantly degrade the stent and markerball signatures. In the application of MCI, x-ray frames associated with significant motion should be discarded. In Figure 4 we present a clinical example of the improved performance achieved with MCI by discarding such frames. 


\section{Conclusion}

Motion Compensated Integration (MCI) improves discrimination between partially deployed and fully deployed stents aiding deployment assessment. Proper deployment is believed important to maximize patient outcome. The required registration accuracy $(\sim 2$ pixels or less) relative to the markerball radius $(\sim 3$ pixels) appears achievable given that markerballs may be reliably detected.

MCI provides measurable, but modest improvement on observer assessment of stent deployment. For MCI to gain wide clinical use requires that it be appropriately integrated into the interventional cardiologist's workflow. This implies near real-time robust unsupervised markerball detection and tracking. Also, contrast enhancement of the displayed MCI image remains to be explored.

\section{References}

1. Heart disease and stroke statistics - 2005 update. Technical report, American Heart Association, Dallas, TX (2004)

2. Koolen, J.J., Ziekenhuis, C.: Drug eluting stents: The importance of deployment. In: EuroPCR. (2003)

3. Hartley, R.I., Zisserman, A.: Multiple View Geometry in Computer Vision. Cambridge University Press, Cambridge, UK (2000)

4. Gilmore, G.C., Xue, P., Thomas, C.W., Wilson, D.L.: An adaptive reference/test paradigm: Application to pulsed fluoroscopy perception. Behav. Res. Methods Instrum. Comput. 30 (1998) 332-348

5. Green, D., Swets, J.A.: Signal Detection Theory and Psychophysics. Krieger, New York (1974)

6. Xue, P., Wilson, D.L.: Detection of moving objects in pulsed-x-ray fluoroscopy. Journal of the Optical Society of America - A 15 (1998) 375-388

7. Whiting, J.S., Eckstein, M.P., Morioka, C.A., Eigler, N.L.: Effect of additive noise, signal contrast, and feature motion on visual detection in structured noise. In Kundel, H.L., ed.: Proc. SPIE Medical Imaging 1996: Image Preception. (1996) $26-38$ 